\title{
Evaluation of the micro-shear bond strength of four adhesive systems to dentin with and without adhesive area limitation
}

\author{
Yuan Chai ${ }^{\mathrm{a}, \mathrm{b}}$, Hong Lin ${ }^{\mathrm{a}, \mathrm{b},{ }^{*}}$ Gang Zheng ${ }^{\mathrm{a}, \mathrm{b}}$, Xuehui Zhang ${ }^{\mathrm{a}, \mathrm{b}}$, Guangliang Niu ${ }^{\mathrm{c}}$ and Qiao Du ${ }^{\mathrm{c}}$ \\ ${ }^{a}$ Department of Dental Materials \& Dental Medical Devices Testing Center, Peking University School \\ and Hospital of Stomatology, Beijing, China \\ ${ }^{b}$ National Engineering Laboratory for Digital and Material Technology of Peking University School \\ and Hospital of Stomatology Beijing, China \\ ${ }^{c}$ Beijing Hospital of Integrated Traditional and Western Medicine, Beijing, China
}

\begin{abstract}
The purpose of this study was to evaluate the bonding ability of four representative dentin-adhesive systems by applying the micro-shear bond strength ( $\mu$-SBS) test method and to evaluate the influence of adhesive area limitation on the bond strength. Two different adhesive application methods were used in the $\mu$-SBS test (with and without adhesives area limitation), and four representative adhesive systems were used in this study. Each dentin surface was treated with one of the four representative adhesive systems, and with twenty samples per group $(n=20)$, each of the four groups underwent a $\mu$-SBS test. The results showed that the bond strength was significantly influenced by the adhesive application method $(p<0.05)$, the adhesive type $(p<0.05)$ and the interaction between the two factors $(p<0.05)$. With regard to the four representative dentinadhesive systems, 3-E\&R has a much better bond quality compared to the other adhesive systems. Furthermore, the microshear bond strength test method of restricting the area of both the adhesive and the resin is more reliable for evaluating the bonding property of adhesives to dentin, and it is also adequate for comparing the different adhesives systems.
\end{abstract}

Keywords: Adhesive area limitation, adhesive system, dentin, adhesive, micro-shear bond strength

\section{Introduction}

Adhesive dentistry has generally revolutionized modern dentistry over the past decades. With continuously improving bonding technology, the adhesive-resin restoration strategy is being used more and more often in clinical practice due to the focus of less invasiveness and esthetic property [1]. Moreover, the long-term clinical success of adhesive restoration is primarily dependent on the bonding quality of adhesive systems to dentin, and the key parameter for evaluating the bond quality of different dentin-adhesives systems is bond strength [2]. However, it is difficult to obtain precise and consistent bond strength data due to the use of numerous testing techniques and parameters [3, 4]. For example, some factors affect testing results, such as different adhesive areas, the crossing speed, the

\footnotetext{
* Address for correspondence: Hong Lin, Department of Dental Materials \& Dental Medical Devices Testing Center, Peking University School and Hospital of Stomatology, No. 22 South Avenue Zhongguancun, Haidian District, Beijing 100081, China. Tel.: +86 010 82195266; Fax: +86 010 62164691; E-mail: hong196lin@sina.com.
} 
selection and preparation of substrate, the direction of shear force, and so on [5, 6]. Therefore, an accurate and unified laboratory evaluation method is crucial for the effective clinical application of different adhesives, and just as important, differences in the testing procedures should be identified and standardized for consistency.

Recently, the micro-shear bond strength ( $\mu$-SBS) test has been advocated as a modified method for evaluating the bonding ability of dentin-adhesive systems [7-9]. Compared to the macro-shear bond strength test, the $\mu$-SBS test is more advantageous; it has fewer internal defects as well as more homogeneous stress distributions at the interface due to the use of smaller specimen [10]. In addition, the $\mu$-SBS test does not require an additional specimen trimming process after the bonding procedure, which conserves the integrity of the specimens and avoids pre-testing failures [11]. However, due to the unrestricted adhesive area, the traditional $\mu$-SBS test technique still does not produce precise data. Traditionally, the adhesive is applied and cured on the entire substrate prior to the construction of the composite cylinder, instead of constraining the adhesive area to the substrate $[12,13]$. More recently, Shimaoka, et al. [14] proposed that the adhesive area should be delimitated and constrained to the dentin substrate so as to equate the area between the adhesive and the resin and to eliminate differences in test results caused by traditional adhesive application technology. Furthermore, constraining the adhesive area has also been proposed to improve the accuracy of test data concerning the tensile bond strength, and it has been concluded that applying the adhesive on the whole dentin and without adhesive area limitation potentially modifies the local stress distribution at the adhesive/dentin interface [15]. However, no study to date has effectively tested the different categories of dentinadhesives systems by applying adhesive area restriction.

In a previous study, an accepted classification of dentin adhesives divided adhesives into two systems: etch-and-rinse and self-etching systems. The classification was based upon whether or not a separate etching agent was used $[16,17]$. An etch-and-rinse system is characterized by removing the smear layer by using an etching agent [18], and a self-etching system is characterized by eliminating the technique-sensitive rinsing step and reducing the operation time [19]. ART Bond (Coltene) and Adper Single Bond 2 (3M ESPE) are products used with etch-and-rinse systems, and they are commonly used in clinics. In contrast, Clearfil SE Bond (Kuraray) and Adper Easy One (3M ESPE) are both employed in self-etching systems and are the dentin-adhesive materials that are most commonly used in daily clinical practice. Furthermore, Clearfil SE Bond (Kuraray) is considered as the golden standard for self-etch adhesive systems, and Adper Easy One (3M ESPE) is a representative material of one-component self-etching adhesives.

Based on the above considerations, the purpose of this study is and to evaluate the bonding ability of the four above classes of dentin adhesives with and without adhesive area limitation and to evaluate the feasibility of adhesive area delimitation as a standardized laboratory examination method.

\section{Experimental method}

\subsection{Tooth preparation}

One hundred and sixty bovine teeth were used as substrates within three months of extraction. The selected bovine excluded the tooth that had caries, tooth discolored and other disordered and had been stored in a $1 \%$ aqueous solution of chloramine-T. The labial side (bonding side) of each embedded tooth was grinded with 120 -grit silicon paper to expose flat approximately $4 \mathrm{~mm}$ diameter of dentin surface, and subsequently, polished the exposed dentin with 600 -grit silicon paper for 20 s to obtain the 
A
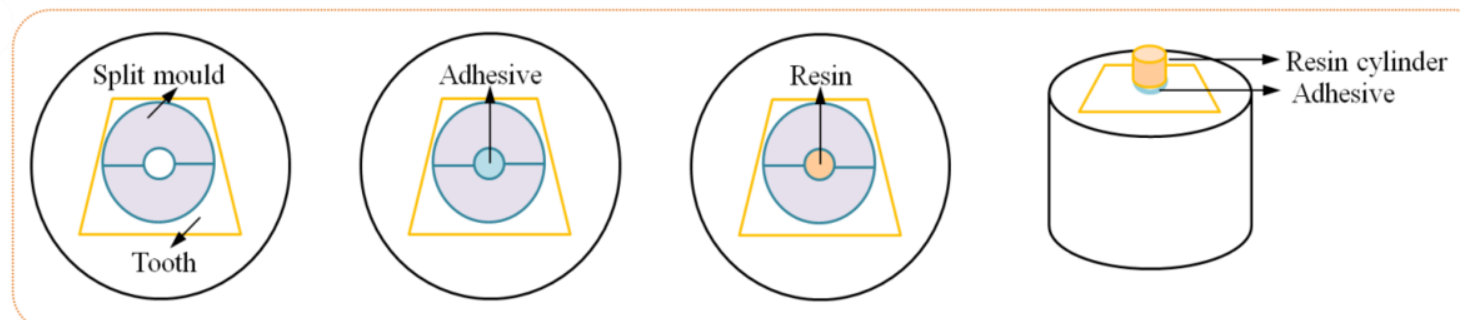

B
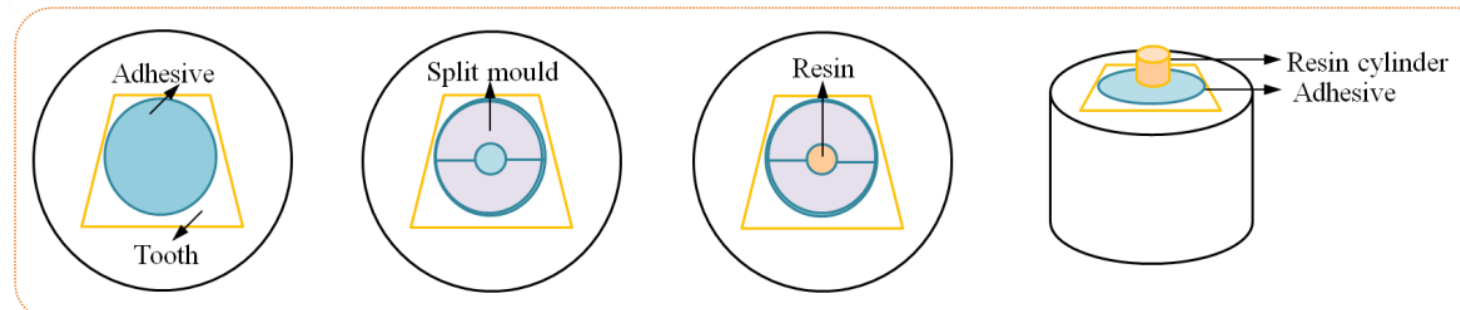

C

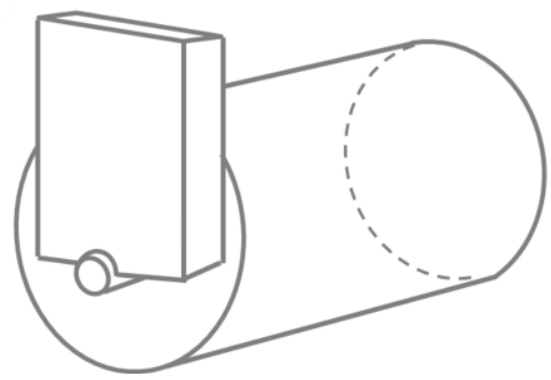

Fig. 1. The two adhesive application methods and the bond strength test apparatus. (A) The method of restricting both the adhesive area and the resin cylinder area. (B) The method of only restricting the resin cylinder area. (C) Diagram of the micro-shear test apparatus.

uniform smear layer.

\subsection{Bonding procedures}

160 prepared bovine teeth were divided into two groups for applying two different adhesive application methods. In Group I, the adhesive area was constrained to the substrate, which first restricted the bonding area by using a $1 \mathrm{~mm}$ diameter PTEE split mold on the dentin, and then, the adhesive and composite were filled and cured, respectively, in this mold (Method I) (Figure 1A). In Group II, the adhesives were applied and cured on the entire dentin instead of the limited adhesive area, and then, the $1 \mathrm{~mm}$ diameter PTEE split mold was used, followed by the construction of the composite cylinder (Method II) (Figure 1B). Each group was then divided into four sub-groups, and each group was treated with one of the four tested adhesives and the corresponding composite resins according to the manufacturers' instructions (Table 1).

\subsection{Micro-shear bond strength test}

Before testing, the diameter of each bonded resin cylinder was measured with an electronic digital 
Table 1

Application of the adhesive materials and composites used in the study

\begin{tabular}{|c|c|c|}
\hline Adhesives & Resins & Application \\
\hline ARTBond (Coltene) & Synergy D6 & Apply etchant for $15 \mathrm{~s} \rightarrow$ rinse and dry $\rightarrow$ apply primer \\
\hline Lot: E91488 & (Coltene) & for $30 \mathrm{~s} \rightarrow$ gently blow with air $\rightarrow$ apply bond for \\
\hline$(3-E \& R)$ & Lot: C11664 & $\begin{array}{l}20 \mathrm{~s} \rightarrow \text { light cure for } 20 \mathrm{~s} \rightarrow \text { place composite } \rightarrow \text { light } \\
\text { cure for } 40 \mathrm{~s}\end{array}$ \\
\hline Adper Single Bond 2 (3M & Filtek Z250 & Apply etchant for $15 \mathrm{~s} \rightarrow$ rinse and dry $\rightarrow$ apply bond $\rightarrow$ \\
\hline ESPE) Lot:M454303 (2- & (3M ESPE) & gently blow with air $\rightarrow$ light cure for $10 \mathrm{~s} \rightarrow$ place \\
\hline $\mathrm{E} \& \mathrm{R})$ & Lot:N377177 & composite $\rightarrow$ light cure for $20 \mathrm{~s}$ \\
\hline Clearfil SE Bond & AP-X (Kuraray) & Apply primer for $20 \mathrm{~s} \rightarrow$ dry $\rightarrow$ Apply bond $\rightarrow$ gently \\
\hline (Kuraray) & Lot:01171A & blow with air $\rightarrow$ light cure for $10 \mathrm{~s} \rightarrow$ place composite $\rightarrow$ \\
\hline Lot:071203 (2-SE) & & light cure for $40 \mathrm{~s}$ \\
\hline $\begin{array}{l}\text { Adper Easy One } \\
(3 \mathrm{M} \text { ESPE) }\end{array}$ & $\begin{array}{l}\text { Filtek Z250 } \\
\text { (3M ESPE) }\end{array}$ & $\begin{array}{l}\text { Apply adhesive for } 20 \mathrm{~s} \rightarrow \text { gently blow with air } \rightarrow \text { light } \\
\text { cure for } 10 \mathrm{~s} \rightarrow \text { place composite } \rightarrow \text { light cure for } 20 \mathrm{~s}\end{array}$ \\
\hline Lot:493837 (1-SE) & Lot:N377177 & \\
\hline
\end{tabular}

caliper (Guanglu, 1-29, China) to confirm the bonding area. The bonded assemblies were immersed in water at $37^{\circ} \mathrm{C}$ for $24 \mathrm{~h}$, and afterwards, they were used for the $\mu$-SBS tests at a crosshead speed of $(1.0$ $\pm 0.1) \mathrm{mm} / \mathrm{min}$ until failure. The direction of the applied force is from the cervical to the incisal site of the tooth (Figure 1C).

\subsection{Mode of failure after micro-shear bond strength tests}

After the $\mu$-SBS tests, all of the de-bonded specimens were observed under a stereo microscope at 40× (Olympics SZX, Japan) and a scanning electron microscope (SEM ZEISS, EV018, Germany) to determine the failure modes. The failure modes were divided into adhesive failure, cohesive failure and mixed failure. The adhesive failure was defined that failure occurred at the dentin/adhesive interface or the adhesive/resin interface. The cohesive failure was defined that the failure occurred at the inside of the resin or dentin. And mixed failure included both adhesive failure and cohesive failure.

\subsection{Statistical analysis}

A two-way ANOVA was conducted to determine the effect of the adhesive application method, adhesive type, and the interaction of the two factors on the bond strength. For each adhesive application method (with and without adhesive area delimitation), one-way ANOVA tests were used to compare the bond strength of the different adhesive types. For all analyses, Tukey's post-hoc test was used for pairwise comparisons. Moreover, the frequency of the failure modes was analyzed by a Chi-square test. Calculations were handled by the software PASW Statistics 17 (SPSS Inc., Chicago, IL, USA), and all of the tests' accuracy was set at a significance level of 0.05 .

\section{Results}

\subsection{Micro-shear bond strength}

Descriptive statistics of the $\mu$-SBS results are shown in Table 2 and Figure 2. The two-way ANOVA 
Table 2

Micro-shear bond strength (Mean $\pm \mathrm{SD}, \mathrm{MPa}$ )

\begin{tabular}{lll}
\hline Adhesives & \multicolumn{3}{l}{ Micro-shear bond strength } \\
\cline { 2 - 3 } & Method I(+) & Method II(-) \\
\hline 3-E\&R & $23.48 \pm 7.57^{\mathrm{Cc}}$ & $29.69 \pm 6.59^{\mathrm{Dd}}$ \\
2- E\&R & $19.07 \pm 5.59^{\mathrm{Aa}}$ & $23.08 \pm 6.51^{\mathrm{Ee}}$ \\
2-SE & $15.07 \pm 5.13^{\mathrm{Bb}}$ & $20.63 \pm 5.96^{\mathrm{Ee}}$ \\
1-SE & $18.74 \pm 5.30^{\mathrm{Aa}}$ & $23.15 \pm 6.63^{\mathrm{Ee}}$ \\
\hline
\end{tabular}

Note: Means followed by the same upper-case letters within any row are not statistically different $(p>0.05)$. Means followed by the same lower-case letters within any column are not statistically different $(p>0.05)$.
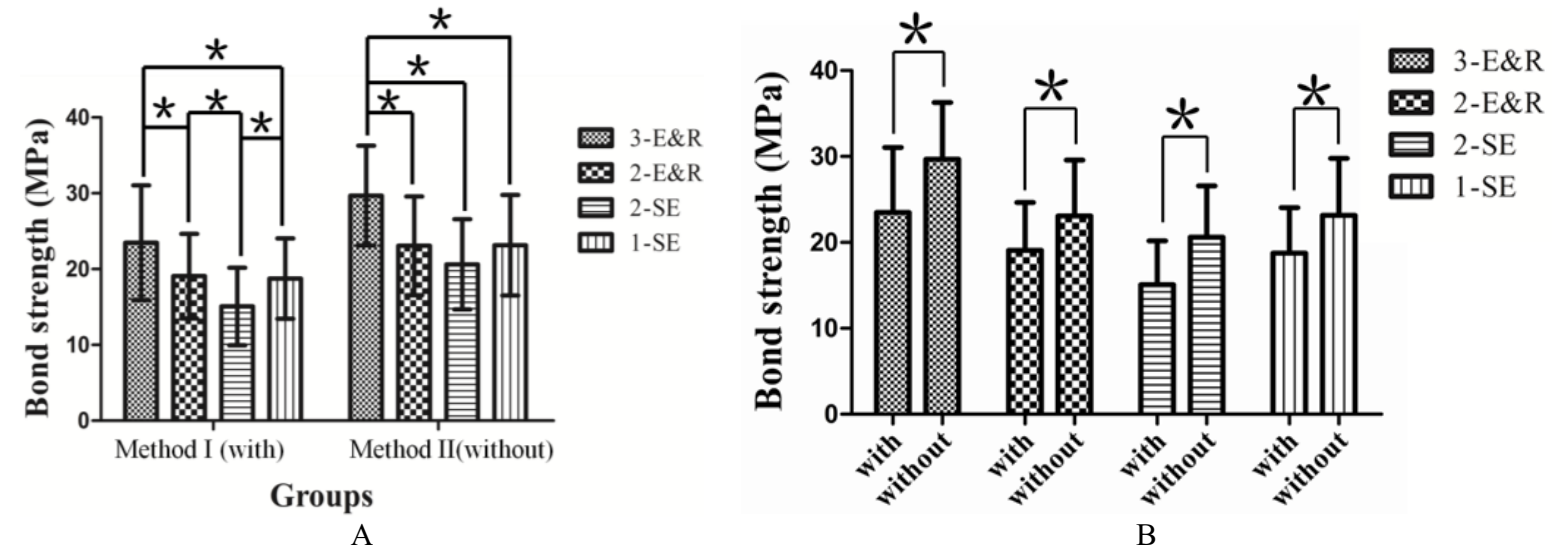

Fig. 2. (A). The micro-shear bond strength of four dentin-adhesive systems with two Methods (with and without adhesive area limitation). Groups with $*$ represent the significant difference of different adhesives. The bond strength can be distinguished with Method I. Only 3-E\&R was observed the significant difference compared to the other adhesives with Method II. (B). The $\mu$-SBS results comparison of a same adhesive with two different adhesive application methods (with and without adhesive area limitation). Groups with $*$ represent the significant difference of $\mu$-SBS test results of an adhesive with two different methods(Tukey's test, $p<0.05$ ). The dentin bond strength of each adhesive without adhesive area limitation distinctly exceeded the corresponding data that was obtained with adhesive area limitation.

indicated that the $\mu$-SBS bond strength was significantly influenced by the adhesive application method, adhesive system type, and the interaction between the two factors $(p<0.05)$. The one-way ANOVA revealed that the $\mu$-SBS test result that was obtained without the adhesive area limitation was significantly higher than the corresponding group with adhesive area limitation, irrespective of the adhesive system types $(p<0.05)$. Significant differences in bond strength were observed when different adhesive systems were applied, and 3-E\&R exhibited the highest bond strength as compared to the other adhesives.

\subsection{Mode of failure observation}

The failure modes of the $\mu$-SBS are shown in Figure 3. The chi-square test of the failure mode frequency of the $\mu$-SBS test specimens indicated that the failure mode frequency was significantly influenced by the adhesive application method for 3-E\&R and 1-SE $(p<0.05)$, whereas there was not a significant difference between the two adhesive application methods for either 2- SE or 2- E\&R. No cohesive failure was observed for Method I (restricting the area of both the adhesive and the resin).

The SEM observation showed that with Method I, the failure geometry was within the area of the 


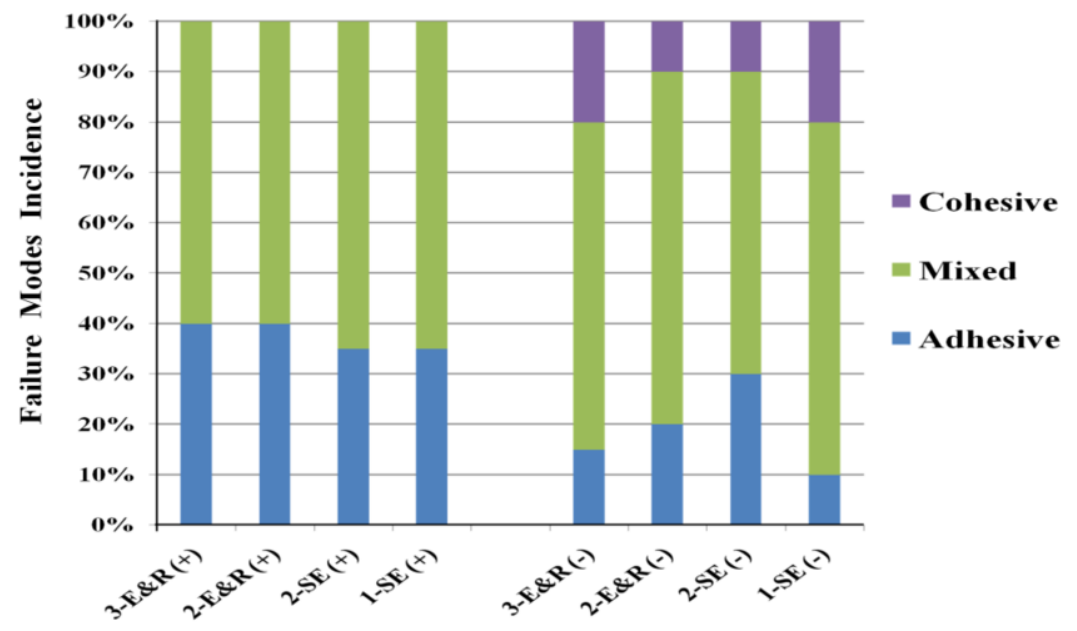

Fig. 3. The failure mode frequency of the $\mu$-SBS test. The Groups with $(+)$ represent the results obtained by the Method I (with adhesive area limitation) and groups with (-) represent the results obtained by Method II (without adhesive area limitation). The groups with Method II express higher cohesive failure percentages.
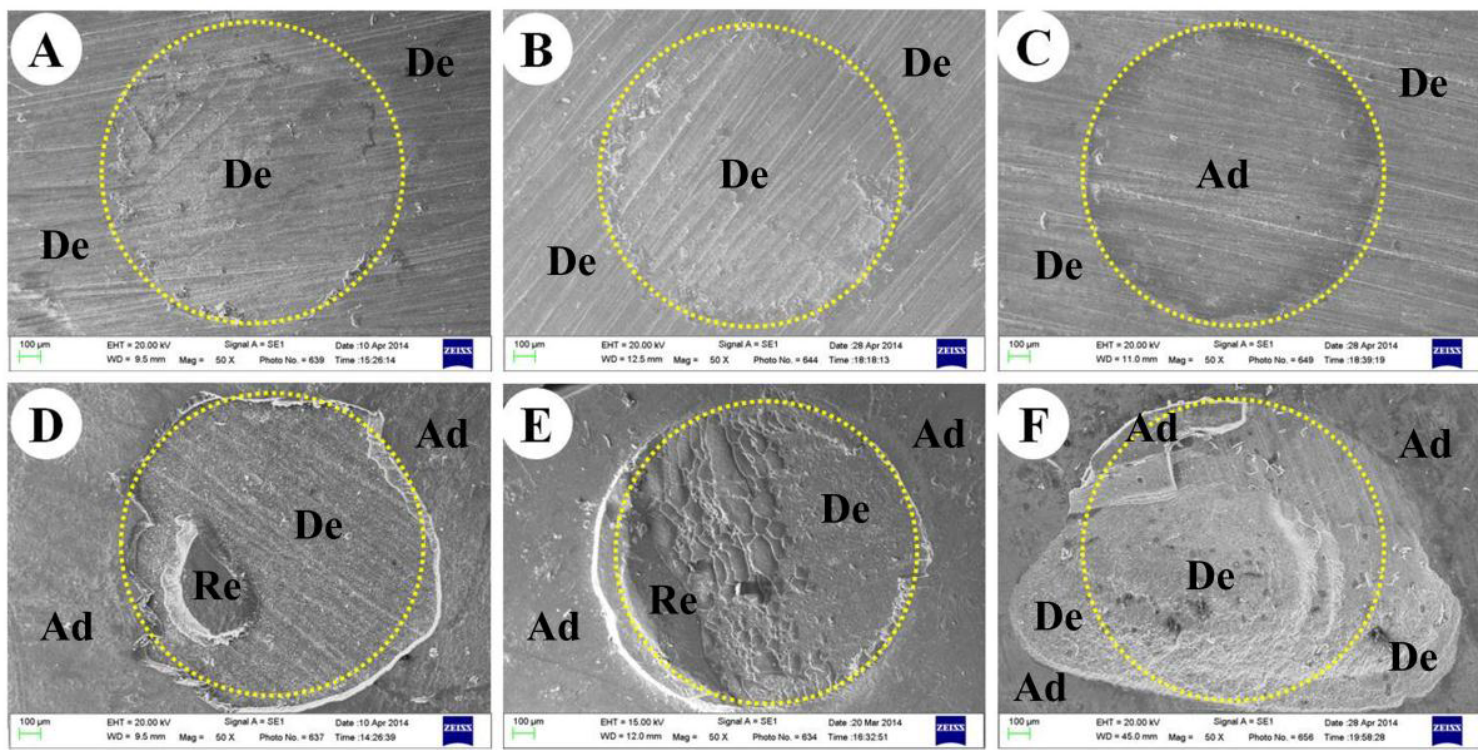

Fig. 4. SEM photomicrographs of the failure modes of the micro-shear test (50x). De: dentin, Ad:adhesive, Re: resin. (A), (B) and (C) are the failure modes with adhesive area limitaiton(restricting both the adhesive and resin cylinder area); (D), (E) and (F) are the failure modes without adhesive area limitaiton(restricting only the resin cylinder area).The dotted loops represent $1 \mathrm{~mm}$ of the resin cylinder area. (A) Adhesive failure occurred at the interface between the dentin and the adhesive. (B) Mixed failure. (C) Adhesive failure occurred at the interface between the adhesive and the resin cylinder. (D) Mixed failure; includes most part of the dentin-adhesive interface failure as well as a small part of cohesive failure in resin. (E) Mixed failure; includes the dentin-adhesive interface failure and the cohesive failure in resin. (F) Mixed failure; includes motly cohesive failure in dentin and only a little adhesive area between the adhesive and the dentin.

adhesive and the resin (Figures 4A-4C). Conversely, with Method II, the failure geometry extended beyond the resin area that was used to calculate the bond strength (Figures 4D-4F). The failure detail of each adhesive system is shown in Figure 5. 

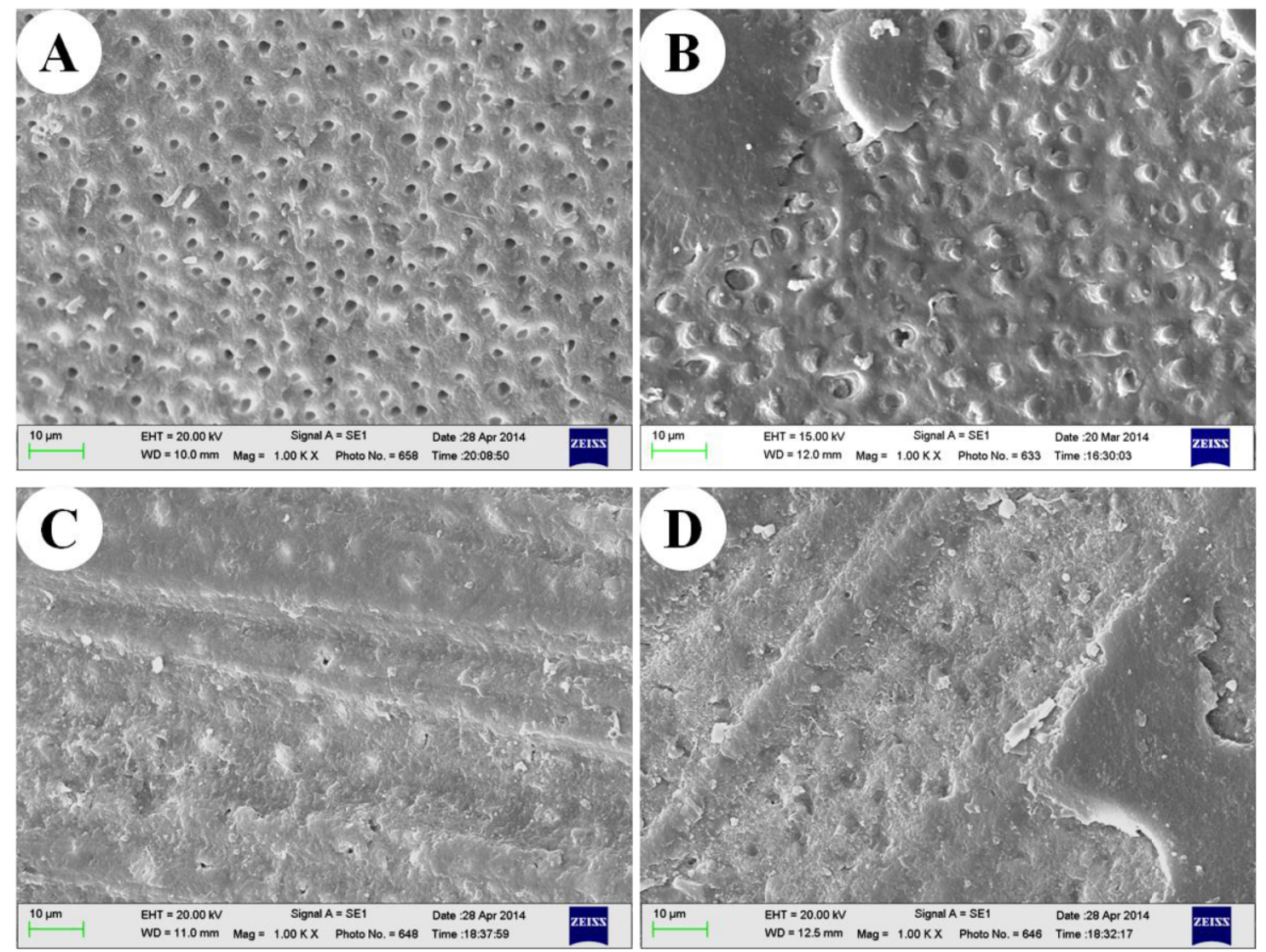

Fig. 5. SEM photomicrographs of the failure modes of the four adhesives (1000x). (A) Cohesive failure in the dentin can be observed in 3-E\&R. The dentinal tubule can be observed clearly, and few resin-tags were observed. (B) The 2-E\&R failure generally occurred at the adhesive/resin interface. The etched dentin was covered with the adhesive and the dentinal tubules were filled with resin tag. (C) The 2-SE failure tended to occur at the dentin/adhesive interface. It is hard to observe the open dentinal tubules. (D) The failure mode of 1-SE tended to occur at the adhesive/resin interface. Mixed failure was observed in $1-\mathrm{SE}$.

\section{Discussion}

The rapid development of adhesive restorative dentistry allows for more conservative and less invasive treatment. In addition, it overcomes the esthetical problems of placing restorations as well as lengthens the lifespan of the teeth. With the development of adhesive restorative dentistry, many new dental adhesive materials were produced. The etch-and-rinse adhesive system and the self-adhesive system are the most frequently employed in clinical works [20]. Before new adhesive materials can be put into clinical use, it must be evaluated through various laboratory methodologies; one such methodology is the $\mu$-SBS test, which is one of the most essential tests for predicting the efficiency of new adhesives [20-22]. However, the reproducibility of testing results is affected by various factors regarding the inherent weakness of test institute [3]. Although ISO 11405 [23] strictly stresses that "a limitation of the bonding area is important," in most $\mu$-SBS tests, the step of restricting the area of both the adhesive and the resin was often ignored [22, 24-27], and only the area of the resin cylinder 
was taken as the bonding area to calculate the bond strength of tested materials. Therefore, the objective of this study was to compare the bond quality of different adhesive systems by employing the $\mu$-SBS test with and without adhesive area restriction. From the results, it was determined that $3 \mathrm{E} \& \mathrm{R}$ has the highest bond strength. In addition, adhesive area limitation is the paramount step for controlling variability in $\mu$-SBS tests.

Restricting the adhesive area in $\mu$-SBS tests is crucial for evaluating and comparing the bonding quality of different dentin-adhesive systems. In this study, restricting the area of both the adhesive and the resin (Method I) and restricting the resin area only (Method II) were respectively applied, and the dentin bond strength of each adhesive without adhesive area limitation distinctly exceeded the corresponding data that was obtained with adhesive area limitation. This is probably due to the difference between the bonding area and the actual tested bonding area with Method II. In the process of the bond strength test, the actual tested bonding area includes not only the area of the resin cylinder but also includes the area of the cured adhesive around the resin cylinder, and the latter was not counted as the bonding test area to calculate bond strength, resulting in the higher nominal bond strength. This outcome was in accordance with R. Van Noort [15] and N. Pecora [28], and the researchers concluded that applying the adhesive on the whole dentin and without adhesive area limitation potentially affects the measurements of dentin tensile or shear bond strength. Moreover, the adhesive property of the different adhesives can be distinguished with Method I (Figure 2), but this is not the case for Method II. In most traditional $\mu$-SBS tests [12, 13, 22, 24-27], the area of the resin cylinder was taken as the bonding area to calculate the bond strength of the tested materials. Therefore, the higher nominal bond strength was obtained and only if massive improvement in bond quality can be distinguished with method II.

The failure mode analysis is considered to be an important parameter for interpreting tests results. In fact, it has been reported that there is a direct positive correlation between the bond strength and cohesive failure [29]. In this study, Method II groups express a higher cohesive failure percentage (Figure 3). From the theoretical aspect, if the typical adhesive failure occurred between the adhesive and the resin cylinder, the bond strength obtained from the two different bonding area restriction methods should always be consistent; however, it is apparent that this testing bond strength cannot represent the bond strength of the adhesive to dentin. When the adhesive failure occurred between the dentin substrate and the adhesive, the bond strength obtained with Method II should obviously surpass the respective data obtained with Method I, and the resultant data obtained with Method II also cannot be identified as the bond strength of this adhesive to dentin. This is primarily because the actual testing area exceeds the bonding area that is calculated with Method II (Figures 4D-4F). In previous studies, the validity of nominal bond strength in $\mu$-SBS tests has been questioned due to a high cohesive failure incidence rate [30]. However the reason for the common occurrence of premature cohesive failure prior to the interface failure is the improved bond strength of modern adhesives as well as the imprecision of bonding area restriction and calculation, which is in line with previous research $[14,15$, 28]. Moreover, R. Van Noort [15] concluded that the stress concentration is situated around the circumference of the resin composite cylinder at the adhesive/resin composite interface when the method mode of adhesive flash is applied by finite element analysis. In this study, the analysis of the failure mode indicates that the cohesive failure rate of the method without adhesive area limitation is higher than the cohesive failure rate of the method with adhesive area limitation.

In addition, two methods (with and without adhesive area limitation) were used in $\mu$-SBS test in this study in order to accurately evaluate and compare the bonding quality of different dentin-adhesive systems. In this study with Method I, the bond strengths of 3-E\&R and 2-E\&R were superior to that of 2 -SE. Furthermore, the SEM image at $1000 \times$ can clearly distinguish the resin-tag that formed 
following the primer/adhesive application on the smear layer-free and demineralized dentine (Figures $5 \mathrm{~A}$ and $5 \mathrm{~B}$ ), and this is perhaps ideal bonding to dentin, which is in agreement with other researchers $[16,20]$. Although the bond strength of 2-SE is lower (possibly because its primmer with high viscosity has an adverse effect on the penetration of resinous monomers into the collagen network leading to the non-ideal hybrid layer formation), there is less adhesive infiltration (Figure 5C) and compromising the bond quality, which is in accordance with some studies [31]. Moreover, wet bonding is very technique-sensitive, and proper water content plays a crucial role in the bonding process. The all-in-one self-etch adhesive system incorporates acid monomers and hydrophilic and hydrophobic components in one bottle. In order to accomplish hard tissue demineralization and resin infiltration simultaneously, more acidic and more hydrophilic counterparts were incorporated, thereby decreasing the technique-sensitivity of wetting bonding.

In this and previous studies, the all-in-one self-etch system achieves acceptable dentin bond strength $[32,33]$, but the durability of the all-in-one self-etch system remains a matter of concern.

\section{Conclusion}

Based on the results of this study, it can be concluded that the adhesive limitation is a vital step for controlling the variability in micro-shear bond strength tests. Furthermore, from the aforementioned results, it was demonstrated that restricting the area of both the adhesive and the resin is a more reliable method for evaluating the bonding property of adhesives to dentin. With regard to the four representative dentin-adhesive systems, 3-E\&R has the superior bond quality compared to the other adhesive systems.

\section{Acknowledgment}

This study was supported by the National Science and Technology support program (No. 2012BAI22B03) of China. All the authors have approved the manuscript, and there are no conflicts of interest to declare.

\section{References}

[1] R.S. Schwartz and R. Fransman, Adhesive dentistry and endodontics: Materials, clinical strategies, and procedures for restoration of access cavities: A review, Journal of Endodontics 31 (2005), 151-165.

[2] K. Sirisha, et al., Validity of bond strength tests: A critical review: Part I, Journal of Conservative Dentistry 17 (2014), 305-311.

[3] S. Armstrong, et al., Adhesion to tooth structure: a critical review of "micro" bond strength test methods, Dental Materials 26 (2014), 50-62.

[4] J. De Munck, et al., Meta-analytical review of parameters involved in dentin bonding, Journal of Dental Research 91 (2012), 351-357.

[5] F.M. El-Askary, et al., Effect of surface area and air-drying distance on shear bond strength of etch-and-rinse adhesive, Brazilian Oral Research 26 (2012), 418-423.

[6] Y. Tamura, et al., Dentin bonding: influence of bonded surface area and crosshead speed on bond strength, Dental Materials Journal 30 (2011), 206-211.

[7] D. Eren, et al., Three different adhesive systems; three different bond strength test methods, Acta Odontologica Scandinavica 71 (2013), 978-983.

[8] W.G. McDonough, et al., A microshear test to measure bond strength of dentin-polymer interfaces, Biomaterials 23 
(2002), 3603-3608.

[9] E.A. Münchow, et al., Microtensile versus microshear bond strength between dental adhesives and the dentin substrate, International Journal of Adhesion and Adhesives 46 (2013), 95-99.

[10] R.R. Braga, et al., Adhesion to tooth structure: A critical review of "macro" test methods, Dental Materials 26 (2010), e38-e49.

[11] A.M. Andrade, et al., Do the microshear test variables affect the bond strength values? International Journal of Dentistry 12 (2012), 1-7.

[12] S.S. Scherrer, et al., Direct comparison of the bond strength results of the different test methods: A critical literature review, Dental Materials 26 (2010), e78-e93.

[13]B. Van Meerbeek, et al., Relationship between bond-strength tests and clinical outcomes, Dental Materials 26 (2010), e100-e121.

[14] A.M. Shimaoka, et al., The importance of adhesive area delimitation in a microshear bond strength experimental design, The Journal of Adhesive Dentistry 13 (2011), 307-314.

[15] R. Van Noort, et al., The effect of local interfacial geometry on the measurement of the tensile bond strength to dentin, Journal of Dental Research 70 (1991), 889-893.

[16]P. Spencer, et al., Adhesive/Dentin interface: The weak link in the composite restoration, Annals of Biomedical Engineering 38 (2010), 1989-2003.

[17] A. Mine, et al., Meta-analytical review of parameters involved in dentin bonding, Journal of Dental Research 91 (2012), 351-357.

[18] N. Manuja, et al., Dental adhesion: mechanism, techniques and durability, The Journal of Clinical Pediatric Dentistry 36 (2012), 223-234.

[19] A. Ishikawa, et al., Micro-tensile and micro-shear bond strengths of current self-etch adhesives to enamel and dentin, American Journal of Dentistry 20 (2007), 161-166.

[20] J. De Munck, et al., Durability of adhesion to tooth tissue: Methods and results, Journal of Dental Research 84 (2005), $18-132$.

[21]E. Placido, et al., Shear versus micro-shear bond strength test: A finite element stress analysis, Dental Materials 23 (2007), 1086-1092.

[22] M. Song, et al., A study on the compatibility between one-bottle dentin adhesives and composite resins using microshear bond strength, Restorative Dentistry and Endodontics 40 (2015), 30-36.

[23] ISO, Dental materials - testing of adhesion to tooth structure, Technical Specification 11405 (2003), 3-7.

[24] A.B. Bavbek, et al., Micro-shear bond strength of adhesive resins to enamel at different relative humidity conditions, Dental Materials Journal 32 (2013), 468-475.

[25] W. Sasakawa, et al., Micro-shear bond strength of five single-steps adhesives to dentin, Dental Materials Journal 24 (2005), 617-627.

[26] A. Tachibana, et al., Influence of blood contamination on bond strength of a self-etching adhesive to dental tissues, The Journal of Adhesive Dentistry 13 (2011), 349-358.

[27] A.G.D.V. Moraesa, et al., Micro-shear bond strength of self-etching systems associated with a hydrophobic resin layer, The Journal of Adhesive Dentistry 13 (2011), 341-348.

[28] P. Yaman, et al., Comparison of shear bond strength relative to two testing devices, Journal of Prosthetic Dentistry 88 (2002), 511-515.

[29] S.R. Armstrong, et al., Mode of failure in the dentin-adhesive resin-resin composite bonded joint as determined by strength-based( $\mu$ TBS) and fracture-based(CNSB) mechanical testing, Dental Materials 17 (2001), 201-210.

[30] A. Versluis, et al., Why do shear bond tests pull out dentin, Journal of Dental Research 76 (1997), 1298-1307.

[31] Y. Liu, et al., Limitations in bonding to dentin and experimental strategies to prevent bond degradation, Dental Materials Journal 90 (2011), 953-968.

[32] C. Harnirattisai, et al., Shear and micro-shear bond strengths of four self-etching adhesives measure dimmediately and 24 hours after application, Dental Materials J 31 (2012), 779-787.

[33]E.L. Pashley, et al., Effects of one versus two applications of an unfilled, all-in-one adhesive on dentine bonding, Journal of Dentistry 30 (2002), 83-90. 